\title{
The role of disease maps in influenza epidemiological surveillance in Japan
}

\author{
Tomohiko Arahori ${ }^{\mathrm{a},}$ * \\ ${ }^{a}$ Graduate Students of Tokyo Metropolitan University Department of Geography, tomohiko.arahori@ gmail.com \\ * Corresponding author
}

Keywords: Disease maps, Epidemiological surveillance, Influenza, Japan

\begin{abstract}
:
Since movement between countries has become easier for people, preventing the spread of various infectious diseases occurring around the world has become an international public health challenge. The use of "disease maps" in infectious disease prevention can encourage a range of studies on patterns of occurrence and modes of transmission to create thematic visual guides charting the geographical spread of diseases, and infectious disease research agencies around the world are conducting epidemiological surveillance for tracking and analyzing the occurrence of infectious diseases. In the case of Japan, influenza is a recurring and widespread infectious disease for which surveillance programs involving mapping are ongoing. With the development of Information-Communication Technology (ICT), surveillance information is easily accessible on the Web to both medical professions and ordinary citizens, as are online Geographical Information Systems (GIS) utilizing these data. Adapting to these transformations in the environment of information delivery requires that disease maps reflect an accurate grasp of local trends and real-time information delivery.
\end{abstract}

Most studies of disease mapping have focused on the technical dimensions of the utilization of disease maps and WebGIS, and very little research has evaluated the use of disease maps and the quality of surveillance information on a regional scale. Accordingly, the aim of this study is to examine the current status of utilization of disease maps in Japan and issues for health crisis management, with a focus on local influenza surveillance.

We conducted a survey of the websites of specialized agencies and local governments in Japan and we engaged in semi-structured interviews with officials from nine agencies that deliver epidemiological information using disease maps. The website survey assessed the quality of information provided by 82 public health institutes, 552 public health centers, 1,042 medical associations, and 1,977 local governments based on an index of 15 items measuring spatial scale along with map forms and usage. Interview items encompassed the "history of construction and management of the local surveillance system," "users and utilization of local surveillance," "impact of introduction and relationship with other surveillance measures," "new developments and enhancements."

From the results of website survey, we found that only 332 agencies and local governments delivered original information on infectious disease jurisdictional districts, namely 116 public health centers, 108 medical associations, and 51 local governments. The spatial scale of surveillance generally corresponded to the jurisdictions of agencies and local governments; however, medical associations are provided at various levels, such as the county and city medical association level, municipal district level, public school district level, chome and aza (block) level, school facility level, and hospital and clinic levels. Among a total of 56 agencies and local governments conducting visualization, only few three were found to be using WebGIS.

The semi-structured interviews revealed that the construction of local surveillance systems was necessary due to limitation of existing nationwide surveillance in rapid information delivery. Specialized agencies and local governments operating regional surveillance pointed out three problems of current nationwide infectious disease surveillance. First, the number of patients reported is limited because a few sentinel medical institutions report this kind of information. Second, use of the FAX information delivery system results in a time lag of two weeks for data aggregation and delivery. Third, because the minimum spatial unit is the public health center level, detailed information at the level under jurisdiction is not available. Such challenges arose amidst the crisis caused by the influenza A/H1N1pdm09 pandemic in 2009. In the aftermath of the failures of the 2009 epidemiological response, agencies and local governments cooperated with local government departments and the board of education to establish a system to collect information from all medical institutions and school facilities in the jurisdiction. Through visualizing such data as a disease map and sharing progress online, local surveillance has been effective in supporting medical consultations among local doctors, infection prevention at school facilities, and emergency response for night-time medical treatment, among other interventions.

Local surveillance is informed by data in the jurisdiction of each specialized agency and local government; however, disease maps utilizing these information are maintained by 56 agencies and local governments. To construct the 
information delivery system on the local scale, we recommend the promotion of cooperation between agencies and local government departments and the strengthening of interactions between medical professionals, and mapping and GIS experts. In addition, it is evident that disease maps are effective for medical consultation support and infection control measures in familiar areas. From this perspective, local-scale disease maps have potential to be utilized as a communication tool for the sharing of risk management information between medical profession and residents. 